\title{
A CHARACTERIZATION OF POTENTIAL SPACES
}

\author{
JOSÉ R. DORRONSORO
}

\begin{abstract}
A mean oscillation characterization, valid for all $\alpha>0$, of the spaces $L_{\alpha}^{p}$ of Bessel potentials of $L^{p}$ functions is given and is used to relate the known characterizations for $0<\alpha<2$ via Marcinkiewicz integrals, due to E. M. Stein, and via vector-valued means of differences, due to R. S. Strichartz.
\end{abstract}

1. Introduction. The Bessel potential of order $\alpha, \alpha>0$, of a smooth function $g$ is defined as $J_{\alpha} g=K_{\alpha} * g$, where

$$
\left(K_{\alpha}\right)^{\Upsilon}(\xi)=\left(1+4 \pi^{2}|\xi|^{2}\right)^{-\alpha / 2} .
$$

The kernel $K_{\alpha}$ can be shown to be integrable and, for $1 \leq p \leq \infty$, the potential spaces $L_{\alpha}^{p}$, introduced by Aronszajn and Smith and by Calderón, are then the spaces of Bessel potentials of order $\alpha$ of $L^{p}$ functions: that is,

$$
L_{\alpha}^{p}=\left\{J_{\alpha} g: g \in L^{p}\right\} .
$$

With the norm $\left\|J_{\alpha} g\right\|_{p, \alpha}=\|g\|_{p}, L_{\alpha}^{p}$ becomes a Banach space.

This paper is concerned with the question of when a given function $f$ is the Bessel potential of an $L^{p}$ function. An obviously necessary condition is that $f$ be in $L^{p}$, and Stein proved [7], for $0<\alpha<2$ and $2 n / n+2 \alpha<p<\infty$, that an $L^{p}$ function $f$ belongs to $L_{\alpha}^{p}$ if and only if its Marcinkiewicz integral

$$
D_{\alpha} f(x)=\left(\int_{\mathbf{R}^{n}}\left|\Delta_{y}^{[\alpha]+1} f(x)\right|^{2}|y|^{-2 \alpha-n} d y\right)^{1 / 2}
$$

is in $L^{p}$ and, furthermore, $\|f\|_{p, \alpha} \sim\|f\|_{p}+\left\|D_{\alpha} f\right\|_{p}$ (by $A \sim B$ we mean there is a constant $C$ independent of $A$ or $B$ such that $C^{-1} A \leq B \leq C A$; also, $\Delta_{y} f(x)=$ $f(x+y)-f(x))$.

With $p$ now such that $1<p<\infty$, the following characterization of Bessel potentials for the same range of $\alpha$ is due to Strichartz [9]. Let $[\alpha]$ denote the integral part of $\alpha$.

THEOREM 1. Let $0<\alpha<2$ and $1<p<\infty$. An $L^{p}$ function $f$ belongs to $L_{\alpha}^{p}$ if and only if the function

$$
S_{\alpha} f(x)=\left(\int_{0}^{\infty}\left(t^{-\alpha} \int_{|y| \leq 1}\left|\Delta_{t y}^{[\alpha]+1} f(x)\right| d y\right)^{2} t^{-1} d t\right)^{1 / 2}
$$

belongs to $L^{p}$. Furthermore, $\|f\|_{p, \alpha} \sim\|f\|_{p}+\left\|S_{\alpha} f\right\|_{p}$.

This result was extended by Bagby $[\mathbf{1}]$ to the range $0<\alpha<n$.

Received by the editors June 8, 1984 and, in revised form, September 21, 1984.

1980 Mathematics Subject Classification. Primary 46E35; Secondary 26A16. 
We will show here how Bessel potentials of $L^{p}$ functions, $1<p<\infty$, can be characterized for a general $\alpha>0$. This will be done by means of a mixed norm estimate of the approximation of these functions by polynomials. To be more precise, let $f$ be a locally integrable function and $Q$ a cube in $\mathbf{R}^{n}$. We denote by $P_{Q}^{k} f$ the unique polynomial of degree $k$ such that

$$
\int_{Q}\left(f(y)-P_{Q}^{k} f(y)\right) y^{\gamma} d y=0
$$

for each $n$-tuple $\gamma=\left(\gamma_{1}, \ldots, \gamma_{n}\right) \in \mathbf{N}^{n}$ such that $|\gamma|=\gamma_{1}+\cdots+\gamma_{n} \leq k$ (for instance, if $k=0, P_{Q}^{0} f$ is just the mean $\left.f_{Q}=|Q|^{-1} \int_{Q} f\right)$. We will write $P_{Q} f$ or even $P_{Q}$ if there is no chance of confusion. Then, for $x \in \mathbf{R}^{n}$ and $t>0$, we define

$$
\Omega_{f}^{k}(x, t)=\Omega_{f}(x, t)=\sup \left\{|Q|^{-1} \int_{Q}\left|f-P_{Q}^{k} f\right|: x \in Q,|Q|=t^{n}\right\} .
$$

The main result of this paper is

THEOREM 2. Let $\alpha>0$ and $f \in L^{p}, 1<p<\infty$. Then $f \in L_{\alpha}^{p}$ if and only if the function

$$
G_{\alpha} f(x)=\left(\int_{0}^{\infty}\left(t^{-\alpha} \Omega_{f}^{[\alpha]}(x, t)\right)^{2} t^{-1} d t\right)^{1 / 2}
$$

is in $L^{p}$. Furthermore, $\|f\|_{p, \alpha} \sim\|f\|_{p}+\left\|G_{\alpha} f\right\|_{p}$.

The paper is organized as follows: $\S 2$ contains the proof of Theorem 2 when $0<\alpha<1$; in view of Strichartz's theorem, we will prove that $G_{\alpha} f \in L^{p}$ if and only if $S_{\alpha} f \in L^{p}$. In $\S 3$ we deal with the case $\alpha>0, \alpha$ nonintegral, by reducing it to that of $\S 2$. Complex interpolation is then used in $\S 4$ to deal with the remaining case $\alpha>0, \alpha$ integral. Finally, $\S 5$ contains the relationship between $G_{\alpha} f$ and the Marcinkiewicz integral $D_{\alpha} f$, and some further results.

2. The case $0<\alpha<1$. We begin with some remarks about the polynomials $P_{Q}$. Let $f$ be a locally integrable function. An easy homogeneity argument gives [6]

$$
\underset{Q}{\operatorname{ess} \sup _{Q}}\left|P_{Q}^{k} f\right| \leq C|Q|^{-1} \int_{Q}|f|,
$$

for any cube $Q$ and $k \geq 0$, and where $C$ does not depend on $Q$ or $f$ (throughout the paper $C$ will denote any absolute constant independent of the particular functions, points or sets considered). As a consequence, for any $n$-tuple $\gamma \in \mathbf{N}^{n}$, we have for the $\gamma$-derivatives of $P_{Q}($ see $[3,4])$

$$
\begin{aligned}
\underset{Q}{\operatorname{ess} \sup }\left|D^{\gamma}\left(P_{Q}^{k} f\right)\right| & \leq C|Q|^{-\gamma / n} \underset{Q}{\operatorname{ess} \sup _{Q}}\left|P_{Q}^{k} f\right| \\
& \leq C|Q|^{-\gamma / n-1} \int_{Q}|f| .
\end{aligned}
$$

It also follows from (1) that, since $P_{Q}^{k}(f+R)=P_{Q}^{k} f+R$ for any polynomial $R$ of degree $\leq k$,

$$
|Q|^{-1} \int_{Q}\left|f-P_{q}^{k} f\right| \leq C|Q|^{-1} \int_{Q}|f-R|
$$


and that if $Q \subset Q^{\prime}$,

$$
|Q|^{-1} \int_{Q}\left|f-P_{Q}^{k} f\right| \leq C\left(\left|Q^{\prime}\right| /|Q|\right)\left|Q^{\prime}\right|^{-1} \int_{Q^{\prime}}\left|f-P_{Q^{\prime}}^{k} f\right| .
$$

The cube with centre $x$ and side length $t$ will be denoted by $Q_{x, t}$. From (4) we obtain

$$
\Omega_{f}^{k}(x, t) \leq C t^{-n} \int_{Q_{x, 2 t}}\left|f-P_{Q_{x, 2 t}}\right|
$$

and therefore, if $t \leq s \leq 2 t, \Omega_{f}^{k}(x, t) \leq C \Omega_{f}^{k}(x, s) \leq C \Omega_{f}^{k}(x, 2 t)$. In particular, the series

$$
\left(\sum_{-\infty}^{\infty}\left(2^{-i \alpha} \Omega_{f}\left(x, 2^{i}\right)\right)^{2}\right)^{1 / 2}
$$

is comparable to $G_{\alpha}$, and we can also use balls instead of cubes to define $\Omega_{f}$ and $G_{\alpha} f$. We have now

THEOREM 3. Let $\alpha>0, \alpha$ nonintegral, and $f \in L^{p}, 1<p<\infty$. Then $G_{\alpha} f \in L^{p}$ if and only if for a.e. $x \in \mathbf{R}^{n}$ there is a polynomial $P_{x}$ of degree $\leq[\alpha]$, depending on $f$, such that if

$$
\tilde{G}_{\alpha} f(x)=\left(\int_{0}^{\infty}\left(t^{-\alpha-n} \int_{Q_{x, t}}\left|f(y)-P_{x}(y)\right| d y\right)^{2} t^{-1} d t\right)^{1 / 2}
$$

$\tilde{G}_{\alpha} f \in L^{p}$. Furthermore, $\left\|G_{\alpha} f\right\|_{p} \sim\left\|\tilde{G}_{\alpha} f\right\|_{p}$. The polynomial $P_{x}$ will be a.e. the Taylor polynomial of $f$.

Proof. The inequality $G_{\alpha} f \leq C \tilde{G}_{\alpha} f$ is an immediate consequence of (5) and (3). Conversely, if $G_{\alpha} f \in L^{p}$, fix $x \in \mathbf{R}^{n}, \gamma \in \mathbf{N}^{n}$ with $|\gamma| \leq[\alpha]$ and write $P_{Q_{x, t}}^{[\alpha]} f(y)=P_{Q_{x, t}}(y)$ as

$$
P_{Q_{x, t}}(y)=\sum_{|\beta| \leq[\alpha]} a_{\beta}(x, t)(y-x)^{\beta} / \beta !
$$

If $t>s>0$, let $Q_{0} \subset Q_{1} \subset \cdots \subset Q_{m}$ be a sequence of cubes such that $Q_{0}=$ $Q_{x, s}, Q_{m}=Q_{x, t}$ and $\left|Q_{i}\right|=2^{n}\left|Q_{i-1}\right|$. Then, by (2),

$$
\begin{aligned}
\left|a_{\gamma}(x, t)-a_{\gamma}(x, s)\right| & =\left|D^{\gamma}\left(P_{Q_{0}}-P_{Q_{m}}\right)(x)\right| \\
& \leq \sum_{1}^{m}\left|D^{\gamma}\left(P_{Q_{i}}-P_{Q_{i-1}}\right)(x)\right| \\
& \leq C \sum_{1}^{m}\left|Q_{i}\right|^{-1-|\gamma| / n} \int_{Q_{i}}\left|f-P_{Q_{i}}\right| \\
& \leq C \int_{s}^{t} \Omega_{f}(x, u) u^{-|\gamma|-1} d u .
\end{aligned}
$$

Thus, if $\alpha>|\gamma|$, Schwartz's inequality gives

$$
\left|a_{\gamma}(x, t)-a_{\gamma}(x, s)\right| \leq C t^{\alpha-|\gamma|} G_{\alpha} f(x) .
$$


In addition, we have

$$
\begin{aligned}
\left|a_{\gamma}(x, t)\right| & \leq\left|a_{\gamma}(x, t)-a_{\gamma}(x, 1)\right|+\left|a_{\gamma}(x, 1)\right| \\
& \leq C\left(G_{\alpha} f(x)+M f(x)\right),
\end{aligned}
$$

with $M$ the Hardy-Littlewood maximal operator. Therefore, as $t$ goes to 0 , the functions $a_{\gamma}(x, t)$ form a Cauchy sequence of $L^{p}$ functions. We denote its $L^{p}$ limit by $f_{\gamma}$, which will also be a.e. the pointwise limit of some subsequence $a_{\gamma}\left(x, t_{j}\right)$. Hence, for a.e. $x$

$$
\begin{aligned}
\left|f_{\gamma}(x)-a_{\gamma}(x, t)\right| & =\lim \left|a_{\gamma}\left(x, t_{j}\right)-a_{\gamma}(x, t)\right| \\
& \leq C \int_{0}^{t} \Omega_{f}(x, u) u^{-|\gamma|-1} d u \leq C t^{\alpha-|\gamma|} G_{\alpha} f(x),
\end{aligned}
$$

and, in particular, $\left\|f_{\gamma}\right\|_{p} \leq\left\|f_{\gamma}-a_{\gamma}(\cdot, 1)\right\|_{p}+\left\|a_{\gamma}(\cdot, 1)\right\|_{p} \leq C\left(\left\|G_{\alpha} f\right\|_{p}+\|f\|_{p}\right)$.

If we now define $P_{x}(y)=\sum_{|\gamma| \leq k} f_{\gamma}(x)(y-x)^{\gamma} / \gamma$ !, we obtain

$$
\begin{aligned}
\int_{Q_{x, t}}\left|f-P_{x}\right| d y & \leq \int_{Q_{x, t}}\left|f-P_{Q_{x, t}}\right| d y+C \sum_{|\gamma| \leq k}\left|a_{\gamma}(x, t)-f_{\gamma}(x)\right| t^{|\gamma|} \\
& \leq C t^{-n}\left(\Omega_{f}(x, t)+\sum_{i=0}^{k} t^{i} \int_{0}^{t} \Omega_{f}(x, u) u^{-i-1} d u\right)
\end{aligned}
$$

Assuming that $k=[\alpha]<\alpha$, Hardy's inequality implies

$$
\tilde{G}_{\alpha} f(x) \leq C G_{\alpha} f(x) \text { for a.e. } x \in \mathbf{R}^{n} \text {. }
$$

In particular, since $a_{0}(x, t)=f_{Q_{x, t}}$ tends to $f(x)$ a.e., it follows that for $0<\alpha<$ $1, \tilde{G}_{\alpha} f(x)=S_{\alpha} f(x)$ a.e., and Theorem 2 is proved in this case.

3. The case $\alpha>0, \alpha$ nonintegral. The following is a well-known result in the theory of potential spaces (see, for instance $[8$, p. 136]).

THEOREM 4. Let $f \in L^{p}, 1<p<\infty$. Then $f \in L_{\alpha}^{p}, \alpha \geq 1$, if and only if $f$ and its weak partials $\partial f / \partial x_{i}$ belong to $L_{\alpha-1}^{p}$. Furthermore, $\|f\|_{p, \alpha} \sim\|f\|_{p, \alpha-1}+$ $\sum_{1}^{n}\left\|\partial f / \partial x_{i}\right\|_{p, \alpha-1}$.

A similar result also holds for $L^{p}$ functions $f$ such that $G_{\alpha} f \in L^{p}$; concretely,

THEOREM 5. Let $f \in L^{p}, 1<p<\infty$. Then, for $\alpha>1, G_{\alpha} f \in L^{p}$ if and only if $G_{\alpha-1} f \in L^{p}$, the weak partials $\partial f / \partial x_{i} \in L^{p}$ and $G_{\alpha-1}\left(\partial f / \partial x_{i}\right) \in L^{p}$. Furthermore,

$$
\|f\|_{p}+\left\|G_{\alpha} f\right\|_{p} \sim\|f\|_{p}+\left\|G_{\alpha-1} f\right\|_{p}+\sum_{1}^{n}\left(\left\|\partial f / \partial x_{i}\right\|_{p}+\left\|G_{\alpha-1}\left(\partial f / \partial x_{i}\right)\right\|_{p}\right) .
$$

ProOF. We begin with the only if part. Suppose first that $f$ is a $C^{\mathbf{1}}$ function such that $f$ and $\partial f / \partial x_{i}$ are in $L^{p}$, and fix $x$ and $t>0$. For each $i=1, \ldots, n$ and $y \in Q_{0, t}$, consider the polynomials

$$
P_{i}(x+y)=\int_{0}^{1} P_{Q_{x, s t}}^{k-1}\left(\partial f / \partial x_{i}\right)(x+s y) d s
$$


with $k=[\alpha]$. Then, by Taylor's formula,

$$
\begin{aligned}
& t^{-n} \int_{Q_{x, t}}\left|f(z)-f(x)-\sum_{1}^{n} P_{i}(z)\left(z_{i}-x_{i}\right)\right| d z \\
& \quad \leq t^{-n} \sum_{1}^{n} \int_{0}^{1} t \int_{Q_{0, t}}\left|\partial f / \partial x_{i}(x+s y)-P_{Q_{x, s t}}^{k-1}\left(\partial f / \partial x_{i}\right)(x+s y)\right| d y d s \\
& \quad \leq C \sum_{1}^{n} t \int_{0}^{1}(s t)^{-n} \int_{Q_{0, s t}}\left|\partial f / \partial x_{i}(x+z)-P_{Q_{x, s t}}^{k-1}(x+z)\right| d z d s
\end{aligned}
$$

which implies

$$
\Omega_{f}^{k}(x, t) \leq C \sum_{1}^{n} \int_{0}^{2 t} \Omega_{\partial f / \partial x_{i}}^{k-1}(x, u) d u .
$$

This estimate can be extended to any $f \in L^{p}$ with weak partials in $L^{p}$ by a standard regularization argument and, in the conditions of the theorem, Hardy's inequality yields

$$
G_{\alpha} f(x) \leq C \sum_{1}^{n} G_{\alpha-1}\left(\partial f / \partial x_{i}\right)(x) .
$$

Next, we will divide the proof of the if part into three lemmas.

LEMMA 1. Let $f \in L^{p}$ such that $G_{\alpha} f \in L^{p}$, and $\gamma \in \mathbf{N}^{n},|\gamma|<\alpha$. Then, with $f_{\gamma}$ as in Theorem $3,\left\|G_{\alpha-|\gamma|} f_{\gamma}\right\|_{p} \leq C\left\|G_{\alpha} f\right\|_{p}$.

Proof. Fix $x \in \mathbf{R}^{n}$ and a cube $Q$ with $x \in Q$ and $|Q|=t^{n}$. If $y \in Q, Q_{y, t} \subset$ $Q_{x, 2 t}$, and by (8) and (2),

$$
\begin{aligned}
\left|f_{\gamma}(y)-D^{\gamma} P_{Q_{x, 2 t}}(y)\right| & \leq\left|f_{\gamma}(y)-a_{\gamma}(y, t)\right|+\left|D^{\gamma}\left(P_{Q_{y, t}}-P_{Q_{x, 2 t}}\right)(y)\right| \\
& \leq C \int_{0}^{t} \Omega_{f}(y, s) s^{-|\gamma|-1} d s+C t^{-|\gamma|} \Omega f(x, 2 t) .
\end{aligned}
$$

Therefore,

$$
\begin{aligned}
\Omega_{f_{\gamma}}(x, t) & \leq C t^{-n} \int_{Q_{x, 2 t}} d y\left(\int_{0}^{t} \Omega_{f}(y, s) s^{-|\gamma|-1} d s\right)+C t^{-|\gamma|} \Omega_{f}(x, 2 t) \\
& \leq C \int_{0}^{t} M\left(\Omega_{f}(\cdot, s)\right)(x) s^{-|\gamma|-1} d s+C t^{-|\gamma|} \Omega_{f}(x, 2 t)
\end{aligned}
$$

where $M$ denotes the Hardy-Littlewood maximal operator. Now, Hardy's inequality gives

$$
G_{\alpha-|\gamma|}\left(f_{\gamma}\right)(x) \leq C\left(G_{\alpha} f(x)+\left(\int_{0}^{\infty}\left(t^{-\alpha} M \Omega_{f}(\cdot, t)\right)(x)^{2} t^{-1} d t\right)^{1 / 2}\right)
$$

and the lemma follows by the Fefferman-Stein theorem on vector-valued maximal operators [5]. 
LEMMA 2. Let $f \in L^{p}$ be such that $G_{\alpha} f \in L^{p}, \alpha>1$. Then the weak partials $\partial f / \partial x_{i}$ exist and coincide with $f_{e_{i}}$, where $e_{i}$ denotes the $n$-tuple with 1 in the ith place and 0 in the others.

ProOF. It is enough to show that $\left(f\left(x+t e_{i}\right)-f(x)\right) / t$ tends to $f_{e_{i}}(x)$ in $L^{p}$ as $t$ goes to 0 . First, we have by (8),

$$
\begin{aligned}
& \left|t^{-1}\left(f\left(x+t e_{i}\right)-f(x)\right)-f_{e_{i}}(x)\right| \\
& \leq t^{-1}\left|f\left(x+t e_{i}\right)-a_{0}\left(x+t e_{i}, 2 t\right)\right|+t^{-1}\left|f(x)-a_{0}(x, 2 t)\right| \\
& \quad+\left|t^{-1}\left(a_{0}\left(x+t e_{i}, 2 t\right)-a_{0}(x, 2 t)\right)-f_{e_{i}}(x)\right| \\
& \leq C t^{\alpha-1}\left(G_{\alpha} f\left(x+t e_{i}\right)+G_{\alpha} f(x)\right) \\
& \quad+\left|t^{-1}\left(a_{0}\left(x+t e_{i}, 2 t\right)-a_{0}(x, 2 t)\right)-f_{e_{i}}(x)\right| .
\end{aligned}
$$

Next, since $a_{0}(y, t)=P_{Q_{y, t}}(y)$,

$$
\begin{aligned}
a_{0}(x & \left.+t e_{i}, 2 t\right)-a_{0}(x, 2 t) \\
& =P_{Q_{x+t e_{i}, 2 t}}\left(x+t e_{i}\right)-P_{Q_{x, 2 t}}\left(x+t e_{i}\right)+P_{Q_{x, 2 t}}\left(x+t e_{i}\right)-P_{Q_{x, 2 t}}(x) \\
& =P_{Q_{x+2 t e_{i}, 2 t}}\left(x+t e_{i}\right)-P_{Q_{x, 2 t}}\left(x+t e_{i}\right)+\sum_{j=1}^{[\alpha]} a_{j e_{i}}(x, 2 t) t^{j} / j !
\end{aligned}
$$

thus,

$$
\begin{aligned}
& \left|t^{-1}\left(a_{0}\left(x+t e_{i}, 2 t\right)-a_{0}(x, 2 t)\right)-f_{e_{i}}(x)\right| \\
& \leq\left|t^{-1}\left(a_{0}\left(x+t e_{i}, 2 t\right)-a_{0}(x, 2 t)\right)-a_{e_{i}}(x, 2 t)\right|+\left|a_{e_{i}}(x, 2 t)-f_{e_{i}}(x)\right| \\
& \leq t^{-1}\left|P_{Q_{x+t e_{i}, 2 t}}\left(x+t e_{i}\right)-P_{Q_{x, 2 t}}\left(x+t e_{i}\right)\right| \\
& +C \sum_{j=2}^{[\alpha]}\left|a_{j e_{i}}(x, 2 t)\right| t^{j-1}+C t^{\alpha-1} G_{\alpha} f(x) \\
& \leq C t^{-1} t^{-n} \int_{Q_{x+t e_{i}, t}}\left|P Q_{x+t e_{i}, 2 t}(y)-P_{Q_{x, 2 t}}(y)\right| d y \\
& +C \sum_{j=2}^{[\alpha]} t^{j-1}\left|a_{j e_{i}}(x, 2 t)\right|+C t^{\alpha-1} G_{\alpha} f(x),
\end{aligned}
$$

and taking $L^{p}$ norms, we obtain

$$
\begin{aligned}
& \left\|t^{-1}\left(f\left(\cdot+t e_{i}\right)-f(\cdot)\right)-f_{e_{i}}(\cdot)\right\|_{p} \\
& \quad \leq C t^{-1}\left\|\Omega_{f}(\cdot, 2 t)\right\|_{p}+C \sum_{j=2}^{[\alpha]} t^{j-1}\left\|a_{j e_{i}}(\cdot, 2 t)\right\|_{p}+C t^{\alpha-1}\left\|G_{\alpha} f\right\|_{p} .
\end{aligned}
$$

But $\left\|\Omega_{f}(\cdot, 2 t)\right\|_{p} \leq C t^{\alpha}\left\|G_{\alpha} f\right\|_{p}$ and, if $j<[\alpha],\left\|a_{j e_{i}}(\cdot, 2 t)\right\|_{p} \leq C\left(\left\|G_{\alpha} f\right\|_{p}+\|f\|_{p}\right)$, by (7), whereas if $j=[\alpha]=\alpha$, an easy modification of (7) gives

$$
\left\|a_{j e_{i}}(\cdot, 2 t)\right\|_{p} \leq C\left(\log t^{-1}\right)\left(\left\|G_{\alpha} f\right\|_{p}+\|f\|_{p}\right)
$$

In any case $\left\|t^{-1}\left(f\left(\cdot,+t e_{i}\right)-f(\cdot)\right)-f_{e_{i}}(\cdot)\right\|_{p}$ tends to 0 as $t$ goes to 0 . 
To finish Theorem 5 we will prove

LEMMA 3. Let $f$ be an $L^{p}$ function such that $G_{\alpha} f \in L^{p}, \alpha>1$. Then $G_{\alpha-1} f \in$ $L^{p}$ and $\left\|G_{\alpha-1} f\right\|_{p} \leq C\left(\left\|G_{\alpha} f\right\|_{p}+\|f\|_{p}\right)$.

ProOF. Let $k=[\alpha]$. We will follow the lines of the proofs of similar facts in $[3]$ or [6] (see also [4]). Fix $x \in \mathbf{R}^{n}$ and $t>0$. For each cube $Q$ containing $x$ and such that $|Q|=t^{n}$, write $P_{Q}^{k} f$ as

$$
P_{Q}^{k} f(y)=\sum_{|\gamma|<k} a_{\gamma}(Q)(y-x)^{\gamma}+\sum_{|\gamma|=k} a_{\gamma}(Q)(y-x)^{\gamma}=R_{Q}(y)+S_{Q}(y) .
$$

Then, by (3),

$$
\begin{aligned}
|Q|^{-1} \int_{Q}\left|f-P_{Q}^{k-1} f\right| & \leq C|Q|^{-1} \int_{Q}\left|f-R_{Q}\right| \\
& \leq C\left(\Omega_{f}^{k}(x, t)+\underset{Q}{\operatorname{essup}}\left|S_{Q}\right|\right) .
\end{aligned}
$$

Denoting by $Q_{i}$ the cube with same centre as $Q$ and side length $2^{i} t,(2)$ implies, for each $|\gamma|=k$,

$$
\left|a_{\gamma}\left(Q_{i}\right)\right| \leq C\left(2^{i} t\right)^{-k}\left|Q_{i}\right|^{-1} \int_{Q_{i}}|f| \leq C\left(2^{i} t\right)^{-k-n / p}\|f\|_{p}
$$

that is, $a_{\gamma}\left(Q_{i}\right)$ tends to 0 as $i$ goes to $\infty$ and, again by (2),

$$
\left|a_{\gamma}(Q)\right| \leq \sum_{i=0}^{\infty}\left|a_{\gamma}\left(Q_{i+1}\right)-a_{\gamma}\left(Q_{i}\right)\right| \leq C \sum_{0}^{\infty}\left(2^{i} t\right)^{-k} \Omega_{f}^{k}\left(x, 2^{i} t\right) .
$$

Therefore, inserting this estimate in (9), we obtain

$$
\Omega_{f}^{k-1}(x, t) \leq C\left(\Omega_{f}^{k}(x, t)+t^{k} \int_{t}^{\infty} \Omega_{f}^{k}(x, s) s^{-k-1} d s\right) .
$$

Now Hardy's inequality gives

$$
\begin{aligned}
G_{\alpha-1} f(x) & \leq C\left(\int_{0}^{\infty}\left(t^{-\alpha+1} \Omega_{f}^{k}(x, t)\right)^{2} t^{-1} d t\right)^{1 / 2} \\
& \leq C\left(\left(\int_{0}^{1}\right)^{1 / 2}\right)+C\left(\left(\int_{1}^{\infty}\right)^{1 / 2}\right) \\
& \leq C\left(\int_{0}^{1}\left(t^{-\alpha} \Omega_{f}^{k}(x, t)\right)^{2} t^{-1} d t\right)^{1 / 2}+C M f(x),
\end{aligned}
$$

and the lemma follows.

Theorems 4 and 5 imply that, if the Theorem 2 holds for a given $\alpha$, it also holds for $\alpha+1$. Therefore, the already proved case $0<\alpha<1$ implies that Theorem 2 is true for all $\alpha>0, \alpha$ nonintegral, and that if we can prove it for $\alpha=1$, it will hold for $\alpha$ integral as well. We do this in the following section. 
4. The case $\alpha$ integral. Let $f$ be a locally integrable function and fix an integer $k$ and a cube $Q$ with side length $t>0$. For any $y \in Q$ and any cube $Q^{\prime}$ such that $y \in Q^{\prime} \subset Q$, let $Q^{\prime}=Q_{0} \subset Q_{1} \subset \cdots \subset Q_{m}=Q$ be a sequence of cubes such that $\left|Q_{i}\right|=2^{n}\left|Q_{i-1}\right|$. By (1) and (4) we have

$$
\left|Q^{\prime}\right|^{-1} \int_{Q^{\prime}}\left|f-P_{Q}^{k} f\right| \leq C \sum_{0}^{m}\left|Q_{i}\right|^{-1} \int_{Q_{i}}\left|f-P_{Q_{i}}\right| \leq C \int_{0}^{t} \Omega_{f}(y, s) s^{-1} d s
$$

which by Lebesgue's differentiation theorem gives, for a.e. $y \in Q$,

$$
\begin{aligned}
\left|f(y)-P_{Q}(y)\right| & =\lim _{Q^{\prime} \rightarrow y}\left|Q^{\prime}\right|^{-1} \int_{Q^{\prime}}\left|f-P_{Q}\right| \\
& \leq C \int_{0}^{t} \Omega_{f}(y, s) s^{-1} d s .
\end{aligned}
$$

Now, the second difference operator $\Delta_{h}^{2}$ annihilates polynomials of degree 1 , and for a.e. $x$ we obtain by $(10)$

$$
\begin{array}{r}
t^{-n} \int_{Q_{0, t}}\left|\Delta_{h}^{2} f(x)\right| d h=t^{-n} \int_{Q_{0, t}}\left|\Delta_{h}^{2}\left(f-P_{Q_{x, 2 t}}^{1}\right)(x)\right| d h \\
\leq C \int_{0}^{2 t} M \Omega_{f}^{1}(\cdot, s)(x) s^{-1} d s
\end{array}
$$

which by Hardy's inequality implies

$$
\begin{aligned}
S_{1} f(x) & =\left(\int_{0}^{\infty}\left(t^{-1} t^{-n} \int_{Q_{0, t}}\left|\Delta_{h}^{2} f(x)\right| d h\right)^{2} t^{-1} d t\right)^{1 / 2} \\
& \leq C\left(\int_{0}^{\infty}\left(t^{-1} M \Omega_{f}^{1}(\cdot, t)(x)\right)^{2} t^{-1} d t\right)^{1 / 2} .
\end{aligned}
$$

Thus, if $G_{1} f \in L^{p}, 1<p<\infty$, the $L^{p}$ boundedness of the vector-valued HardyLittlewood maximal operator gives that $\left\|S_{1} f\right\|_{p} \leq C\left\|G_{1} f\right\|_{p}$.

Conversely, to prove that $f \in L_{1}^{p}$ implies $G_{1} f \in L^{p}$, we will use complex interpolation. If $(A, B)_{[\theta]}$ denotes the intermediate space between the Banach spaces $A, B$ obtained by the complex method for the index $\theta$, it is well known (see, for instance [2]) that $\left(L_{\alpha_{1}}^{p}, L_{\alpha_{2}}^{p}\right)_{[\theta]}=L_{\alpha}^{p}$, with $\alpha=\theta \alpha_{1}+(1-\theta) \alpha_{2}$. Suppose now that $0<\alpha_{1}<1<\alpha_{2}<2$ and let $H_{\alpha_{i}}, i=1,2$, denote the Banach space of functions $F(y, t)$ defined in the product $(0, \infty) \times Q_{0,1}$ such that

$$
\|F\|_{H_{\alpha_{i}}}=\left(\int_{0}^{\infty}\left(t^{-\alpha_{i}} \int_{Q_{0,1}}|F(y, t)| d y\right)^{2} t^{-1} d t\right)^{1 / 2}<\infty
$$

By the results in $\S \S 2$ and 3 and the inequality $\Omega_{f}^{1} \leq C \Omega_{f}^{0}$ derived from (4), the map

$$
f \rightarrow F(y, t ; x)=f(x+t y)-P_{Q_{x, t}}^{1} f(x+t y)
$$

is a bounded linear map from $L_{\alpha_{i}}^{p}$ to the $L^{p}$ space of $H_{\alpha_{i}}$ valued functions $L^{p}\left(H_{\alpha_{i}}\right)$ and, therefore, from $L_{1}^{p}=\left(L_{\alpha_{1}}^{p}, L_{\alpha_{2}}^{p}\right)_{[\theta]}$ into $\left(L^{p}\left(H_{\alpha_{1}}\right), L^{p}\left(H_{\alpha_{2}}\right)\right)_{[\theta]}$ with $\theta=$ 
$\alpha_{2}-1 / \alpha_{2}-\alpha_{1}$. But $\left(L^{p}\left(H_{\alpha_{1}}\right), L^{p}\left(H_{\alpha_{2}}\right)\right)_{[\theta]}=L^{p}\left(\left(H_{\alpha_{1}}, H_{\alpha_{2}}\right)_{[\theta]}\right)$ and $\left(H_{\alpha_{1}}, H_{\alpha_{2}}\right)_{[\theta]}=$ $H_{1}\left[2\right.$, pp. 107 and 122]. Therefore, if $f \in L_{1}^{p}$,

$$
\left\|G_{1} f\right\|_{p} \leq C\left\|\left(\|F(\cdot, \cdot ; x)\|_{H_{1}}\right)\right\|_{p} \leq C\|f\|_{p, 1} .
$$

This concludes the proof of Theorem 2 when $\alpha=1$ and, by the preceding remarks, for all $\alpha>0$.

5. Further results. First, we will show how Theorem 2 relates with the Marcinkiewicz integral characterization of $L_{\alpha}^{p}$ when $p>2 n / n+2 \alpha$. For simplicity, we will give the details only for $0<\alpha<1$. In this range of $\alpha$, the Marcinkiewicz integral of a function $f$ can be written as

$$
\begin{aligned}
\left(D_{\alpha} f(x)\right)^{2} & =\int_{\mathbf{R}^{n}}|f(x+y)-f(x)|^{2}|y|^{-2 \alpha-n} d y \\
& =\int_{\mathbf{R}^{n}}|f(x+y)-f(x)|^{2} \int_{|y|}^{\infty} t^{-2 \alpha-n-1} d t d y \\
& =\int_{0}^{\infty} t^{-2 \alpha-n}\left(\int_{|y| \leq t}|f(x+y)-f(x)|^{2} d y\right) t^{-1} d t,
\end{aligned}
$$

which can be seen as a variant of $G_{\alpha} f$. This suggests the possibility of replacing in the definition of $G_{\alpha}$ the $L^{1}$ means $\Omega_{f}(x, t)$ by the $L^{r}$ means

$$
\Omega_{f, r}(x, t)=\sup \left\{\left(|Q|^{-1} \int_{Q}\left|f-P_{Q}\right|^{r}\right)^{1 / r}: x \in Q,|Q|=t^{n}\right\} .
$$

The following theorem tells us when this can be done.

THEOREM 6. Let $\alpha>0$ and $f$ be an $L^{p}$ function, $1<p<\infty$, such that $G_{\alpha} f \in L^{p}$. Then

(i) if $1<p \leq 2$ and $\alpha \leq n / p$, the function $G_{\alpha, r} f$ obtained by replacing $\Omega_{f}$ with $\Omega_{f, r}$ in the definition of $G_{\alpha} f$ is also in $L^{p}$ for $r<p n / n-p \alpha$; if $\alpha>n / p, G_{\alpha, \infty} \in$ $L^{p}$;

(ii) if $2 \leq p<\infty$ and $\alpha \leq n / 2, G_{\alpha, r} f \in L^{p}$ for $r<2 n / n-2 \alpha$; if $\alpha>$ $n / 2, G_{\alpha, \infty} f \in L^{p}$.

Finally, in all cases $\left\|G_{\alpha, r} f\right\|_{p} \sim\left\|G_{\alpha} f\right\|_{p}$.

ProOF. Fix $x \in \mathbf{R}^{n}, t>0$ and a cube $Q$ such that $x \in Q,|Q|=t^{n}$. By (10) we have a.e. in $Q$

$$
\begin{aligned}
\left|f(y)-P_{Q}(y)\right| & \leq C \int_{0}^{t} \Omega_{f}(y, s) s^{-1} d s \\
& \leq C \int_{0}^{t}\left(s^{-n} \int_{Q_{y, s}} \Omega_{f}(z, s) d z\right) s^{-1} d s .
\end{aligned}
$$

Suppose now $1<p \leq 2$ and $\alpha \leq n / p$, and for $r<p n / n-p \alpha$, fix $q<p$ and $\beta<\alpha$ such that $1 / r=1 / q-\beta / n$. By (11),

$$
\left|f(y)-P_{Q}(y)\right| \leq C \int_{0}^{t} s^{-\beta} M_{\beta}\left(\Omega_{f}(\cdot, s) \chi_{Q}\right)(y) s^{-1} d s,
$$


where $M_{\beta}$ denotes the maximal operator $M_{\beta} g(z)=\sup \left\{|Q|^{-1+\beta / n} \int_{Q}|g|: z \in Q\right\}$. As it is well known, $M_{\beta}$ maps $L^{q}$ into $L^{r}$ and, therefore,

$$
\begin{aligned}
\left(\int_{Q}\left|f-P_{Q}\right|^{r}\right)^{1 / r} & \leq C \int_{0}^{t} s^{-\beta}\left(\int_{Q}\left(M_{\beta}\left(\Omega_{f}(\cdot, s) \chi_{Q}\right)\right)^{r} d y\right)^{1 / r} s^{-1} d s \\
& \leq C \int_{0}^{t} s^{-\beta}\left(\int_{Q} \Omega_{f}(z, s)^{q} d z\right)^{1 / q} s^{-1} d s
\end{aligned}
$$

which implies

$$
\Omega_{f, r}(x, t) \leq C t^{\beta} \int_{0}^{t} s^{-\beta} M_{q} \Omega_{f}(\cdot, s)(x) s^{-1} d s,
$$

with $M_{q}(g)=\left(M\left(|g|^{q}\right)\right)^{1 / q}$. Hardy's inequality gives now

$$
G_{\alpha, r} f(x) \leq C\left(\int_{0}^{\infty}\left(t^{-\alpha} M_{q}\left(\Omega_{f}(\cdot, t)\right)(x)\right)^{2} t^{-1} d t\right)^{1 / 2}
$$

and the estimate $\left\|G_{\alpha, r} f\right\|_{p} \leq C\left\|G_{\alpha} f\right\|_{p}$ follows from the Fefferman-Stein theorem. The same proof works for the case $\alpha \leq n / 2$ of (ii): taking $r<2 n / n-2 \alpha$ allows us to choose $q<2$, and to apply the Fefferman-Stein theorem again.

If $p \leq 2$ and $\alpha>n / p$, fix $\beta$ and $q$ such that $\alpha>\beta>n / q>n / p$. From (11) we deduce for a.e. $y \in Q$

$$
\begin{aligned}
\left|f(y)-P_{Q}(y)\right| & \leq C \int_{0}^{t} s^{\beta-n / q} s^{-\beta}\left(\int_{Q_{y, s}} \Omega_{f}(z, s)^{q} d t\right)^{1 / q} s^{-1} d s \\
& \leq C t^{\beta-n / q} \int_{0}^{t} s^{-\beta}\left(\int_{Q} \Omega_{f}(z, s)^{q} d z\right)^{1 / q} s^{-1} d s \\
& \leq C t^{\beta} \int_{0}^{t} s^{-\beta} M_{q} \Omega_{f}(\cdot, s)(x) s^{-1} d s
\end{aligned}
$$

and the inequality $\left\|G_{\alpha, \infty} f\right\|_{p} \leq C\left\|G_{\alpha} f\right\|_{p}$ follows as before. The sarne argument works for the case $\alpha>n / 2$ of (ii). Since the estimate $\left\|G_{\alpha} f\right\|_{p} \leq C\left\|G_{\alpha, r} f\right\|_{p}$ is obvious, Theorem 6 is proved.

As a consequence, $G_{\alpha, 2} f$ characterizes $L_{\alpha}^{p}$ for $p>2 n / n+2 \alpha$ and, since $D_{\alpha} f \sim$ $G_{\alpha, 2} f$, so does $D_{\alpha} f$.

Finally, the characterization of potential spaces in terms of the function $G_{\alpha}$ provides easy proofs of the well-known imbeddings $L_{\alpha}^{p} \subset L_{\beta}^{q}, 1 / p-\alpha / n=1 / q-\beta / n$, and can be used to show that, for $\alpha>n / p, L_{\alpha}^{p}$ is an algebra under multiplication. Also, Besov spaces $B_{\alpha}^{p, q}$ of $L^{p}$ functions (see [8] for the definitions) can be characterized by the finiteness of the expression

$$
\left(\int_{0}^{\infty}\left(t^{-\alpha}\left\|\Omega_{f}^{[\alpha]}(\cdot, t)\right\|_{p}\right)^{q} t^{-1} d t\right)^{1 / q}
$$

Combining this with Theorem 2, easy proofs of the imbeddings $B_{\alpha}^{p, p} \subset L_{\alpha}^{p} \subset$ $B_{\alpha}^{p, 2}, 1<p \leq 2, B_{\alpha}^{p, 2} \subset L_{\alpha}^{p} \subset B_{\alpha}^{p, p}, 2 \leq p<\infty$ and $B_{\alpha}^{p, \infty} \subset L_{\alpha-\varepsilon}^{p}$ for all $\varepsilon>0$ follow. 


\section{REFERENCES}

1. R. J. Bagby, A characterization of Riesz potentials and an inversion formula, Indiana Univ. Math. J. 29 (1980), 581-595.

2. J. Bergh and J. Löfström, Interpolation spaces: An introduction, Springer-Verlag, Berlin, 1976.

3. S. Campanato, Proprieta di una famiglia di spazi funzionali, Ann. Scuola Norm. Sup. Pisa Cl. Sci. (4) 18 (1964), 137-160.

4. R. De Vore and R. Sharpley, Maximal operators and smoothness, Mem. Amer. Math. Soc. No. 293 (1984).

5. C. Fefferman and E. M. Stein, Some maximal inequalities, Amer. J. Math. 93 (1971), 107-115.

6. S. Janson, M. Taibleson and G. Weiss, Elementary characterizations of Morrey-Campanato spaces, Lecture Notes in Math., vol. 992, Springer-Verlag, 1983, pp. 101-114.

7. E. M. Stein, The characterization of functions arising as potentials, Bull. Amer. Math. Soc. 67 (1961), 102-104.

8. __ Singular integrals and differentiability properties of functions, Princeton Univ. Press, Princeton, N.J., 1970.

9. R. S. Strichartz, Multipliers on fractional Sobolev spaces, J. Math. Mech. 16 (1967), 1031-1061.

Divisíon de Matematicas, Facultad de Cíencias, Universidad Autonoma, 28049 MADRID, SPAIN 